\title{
Electrostatic Force Microscopy: Measuring Ion Mobility, Non-linear Optical Signals and Achieving Ultimate Time Resolution
}

Peter Grutter

Department of Physics, McGill University, Montreal, Quebec, Canada

In electrostatic force microscopy (EFM), the electrostatic forces between a tip and a sample are measured with high spatial resolution. This allows sample properties such as ion mobility, electron energy levels of quantum dots and molecules or the interaction of light with matter to be measured with nanometer spatial resolution using techniques of atomic force microscopy (AFM).

\section{Electron spectroscopy by AFM}

The ground and excited electronic level structure of quantum systems is of fundamental importance for their optical, electronic and chemical properties. Single-electron sensitive electrostatic force measurement with AFM has been demonstrated to be capable of quantitative energy level spectroscopy of individual and coupled semiconductor quantum dots (QD). In our experiments the oscillation of a dc biased AFM tip modulates the charge state of a QD. The QD is separated from a back gate via a tunnelling barrier. The resulting charge dynamics leads to measurable changes in cantilever resonance frequency and dissipation. The tip of our AFM can thus be described as a movable gate to address a QD of choice and is at the same time a charge detector with single electron sensitivity. Key to the experimental implementation is that the QD-back electrode tunnelling barrier is engineered to have a QD-back electrode tunnelling rate similar to the AFM cantilever mechanical resonance frequency.

The changes in dissipation and frequency measured by AFM are quantitatively described by the backaction of the single electron on the capacitive coupled AFM tip. We find that the ratio of the measured frequency shift to dissipation directly yields the QD-back electrode tunnelling rate. This allows an experimental determination of the energy dependence of single electron tunnelling rates, yielding quantitative information on the continuous DOS of gold nanoparticles, or the discrete degenerate energy levels in single and coupled InAs QDs [1,2]. Most recently, we have observed quantized electron transfer events at a single molecule-metallic electrode interface using this technique [3]. From these measurements, we can quantitatively extract the electron-nuclear coupling strength, quantized nuclear state transitions, and single-mode phonon-mode reorganization energy upon heterogeneous electron transfer from purely experimental data. Specifically, we can directly measure the lowest energy molecular vibration.

(Collaborators A. Roy-Gobeil, Y. Miyahara, and K. Bevan)

\section{What limits time resolution in AFM?}

Conventional wisdom states that mechanical cantilever frequencies set a limit - miniaturization down to the nanoscale with the associated higher mechanical frequencies are thus the conventional proposed solution. This is correct only for single shot measurements; many interesting ultra-fast phenomena can however be time resolved by using suitable pump-probe techniques that only need relatively 'slow' detectors. Observing ultrafast events combined with nanometer spatial resolution is of great interest in many fields and has been a primary pursuit of multiple research groups [4-6]. I will discuss how time resolution substantially faster than the cantilever period can be achieved. 
First, I will focus on electrical pump-probe techniques by discussing measurements of ion mobility in battery cathodes or the non-equilibrium barriers determining mobility of oxygen vacancies in $\mathrm{SrTiO} 3$ [7, 8].

In particular, I will present in detail how Li ion mobility can be measured by AFM. A major issue inhibiting wide spread deployment is low charging rates of $\mathrm{Li}$ ion batteries. This is related to the poor current understanding of what determines mobility of Li ions in cathode materials. We have used a newly developed electrostatic force microscopy technique to spatially determine variations in Li transport mechanism in LiFePO4, a model cathode material. We applied voltage pulses to the sample and observed the resultant fast time decay of the electrostatic forces due to the mobility of Li ions using a time averaging technique. By performing these experiments as a function of temperature we obtain spatially resolved activation barriers for Li transport. By combining our time resolved AFM techniques with SEM, TOFSIMS and EBSD as well as comparison to DFT calculations we show that ionic transport in these materials must be regarded as a collective effect due to the significant contributions by ion-ion and ion-polaron interactions to the measured activation energies.

(Collaborators A. Mascaro, Z. Wang, P. Hovington, Y. Miyahara, A. Paollela, V. Gariepy, Z. Feng,T. Enright, C. Aiken, K. Zaghib, K. Bevan)

\section{Detecting non-linear optical signals by AFM:}

Optical pump-probe techniques allow for much faster time resolution than electrical pump-probe AFM [4-6]. I will present how the 1ps photocarrier decay time on low-T grown GaAs can be measured using nc-AFM at room temperature [6]. These methods can also be used to spatially resolve the nonlinear optical response to femtosecond laser pump and probe pulses in MoSe2 and lithium niobate, allowing us to measure ultrafast non-linear polarization effects in these samples [9]. We can directly measure a sample response on a 100 fs scale with $10 \mathrm{~nm}$ spatial resolution! Time resolution is only limited by the thermal noise of the AFM detector and thus practically by the properties of our current pump-probe excitation system (i.e. laser pulse length and jitter). In fact, the pump-probe autocorrelation function locally detected by AFM shows a measurable signal difference for delay times between the pump and probe as small as 25as!

The realization of these ultrafast AFM techniques open the door to understanding ultrafast electron dynamics on surfaces, such as the new territory of atomic scale dynamics of polarization or charge transfer events in organic systems.

(Collaborators Z. Schumacher, R. Rejali, R. Pachlatko, A. Spielhofer, Y. Miyahara, D. Cook) 

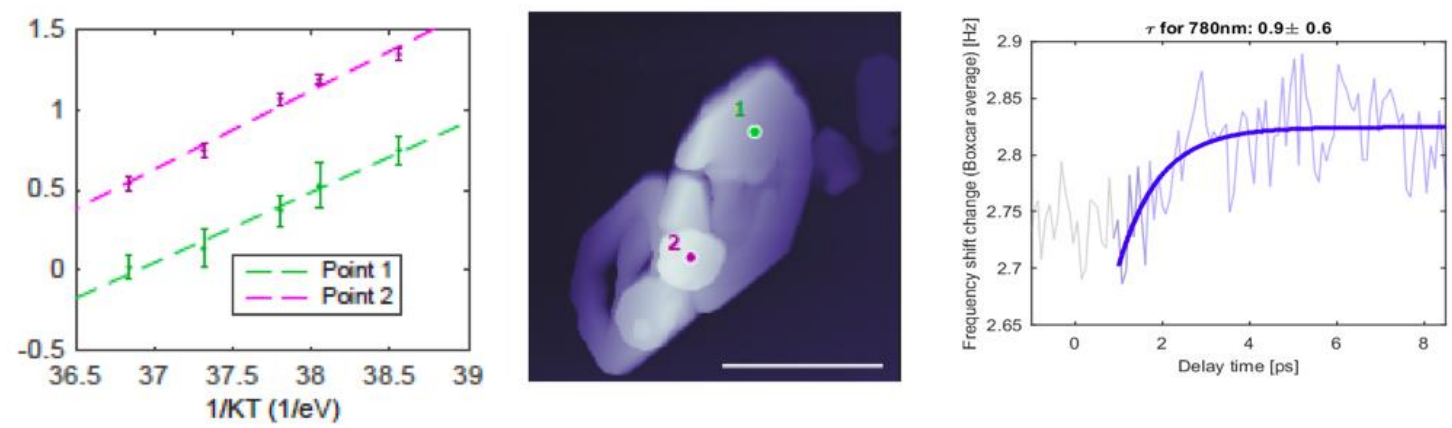

Figure 1. left and center: Spatially resolved activation energy measured on $\mathrm{LiFePO}_{4}$ platelet (scale bar 2 $\mu \mathrm{m})$.right: Photocarrier decay time of 1 ps measured by AFM on LT-GaAs using a fs pump-probe system.

\section{References}

[1] Revealing energy level structure by tunneling rate measured by single-electron sensitive electrostatic force spectroscopy

A. Roy-Gobeil, Y. Miyahara, and P. Grutter,

Nano Lett.15, 2324 (2015)

[2] For a recent review see:

Quantum state readout of individual quantum dots by electrostatic force detection

Y. Miyahara, A. Roy-Gobeil and Peter Grutter,

Nanotechnology 28, 064001 (2017)

[3] Fully Quantum Mechanical Electron Transfer Observed in a Single Redox Molecule at a Metal Interface

A. Roy-Gobeil, Y. Miyahara, K.H. Bevan, and P.Grutter,

Nano Lett. 19, 6104 (2019)

[4] Minority carrier lifetime in polycrystalline silicon solar cells studied by photoassisted Kelvin probe force microscopy

M. Takihara, T. Takahashi, T. Ujihara,

Appl. Phys. Lett. 93, 021902 (2008)

[5] Intensity-Modulated Scanning Kelvin Probe Microscopy for Probing Recombination in Organic Photovoltaics

G. Shao, M. S. Glaz, F. Ma, H. Ju, D. S. Ginger,

ACS Nano 8, 10799 (2014)

[6] The lower limit for time resolution in fm-AFM

Z. Schumacher, A. Spielhofer, Y. Miyahara, P. Grutter,

Appl. Phys. Lett. 110, 053111 (2017)

[7] Collective ionic transport phenomena in lithium battery cathodes: linking experiment and theory

A. Mascaro, Z. Wang, P. Hovington, Y. Miyahara, A. Paolella, V. Gariepy, Z. Feng, T. Enright, C. Aiken, K. Zaghib, K.H. Bevan,

and P. Grutter

Nano Lett. 17, 4489 (2017)

[8] for a recent review see:

Review of time-resolved non-contact electrostatic force microscopy techniques with applications to ionic transport measurements

A. Mascaro, Y. Miyahara, T. Enright, O.E. Dagdeviren, and P. Grutter 
Beilstein J. Nanotechnology 10, 617-633 (2019)

[9] Nanoscale force sensing of ultrafast light induced polarization in dielectrics

Z. Schumacher, R. Rejali, R. Pachlatko, A. Spielhofer, Y. Miyahara, D.G. Cooke, and P. Grutter under review 